International Mathematical Forum, 2, 2007, no. 67, 3323 - 3329

\title{
Three-Step Methods for Nonexpansive Mappings and Mixed Variational Inequalities
}

\author{
Ting Wang ${ }^{1}$, Lin Lin and Yali Zhao ${ }^{2}$ \\ Department of Mathematics, Bohai University \\ Jinzhou, Liaoning 121013, P. R. China
}

\begin{abstract}
In this paper, we suggest and analyze three-step iteration methods for finding the common element of the set of fixed points of nonexpansive mappings and the set of solutions of the mixed variational inequalities. We also study the convergence criteria of three-step iterative method under some mild conditions. Our results are new and interesting and include the previous results as special cases.
\end{abstract}

Keywords: Three-step iteration methods; Common elements; Nonexpansive mappings; Relaxed $(\gamma, r)$-cocoercive mappings; Mixed variational inequalities; Hilbert spaces

\section{Introduction}

Variational inequalities introduced in the early sixties have witnessed an explosive growth in theoretical advances, algorithmic development and applications across all the discipline of pure and applied sciences, see [1-9] and the references therein. It combines novel theoretical and algorithmic advances with new domain of application. Analysis of these problems requires a blend of techniques from convex analysis, functional analysis and numerical analysis. As a result of interaction between different branches of mathematical and engineering sciences, we now have variety of techniques to suggest and analyze various algorithms for solving variational inequalities and related optimization problems. It is well known that variational inequalities are equivalent to the fixed point problems. This alternative equivalent formulation has played a major role in variational inequalities. Recently, Noor and Huang [1] suggested a three-step iterative methods for finding common elements of the fixed points of the nonexpansive mappings and the set of solutions of nonlinear variational inequalities

\footnotetext{
${ }^{1}$ wangting19830826@126.com

${ }^{2}$ Corresponding author. e-mail: yalizhao2000@yahoo.com.cn
} 
by projection technique. On the other hand, mixed variational inequalities are extensions of nonlinear variational inequalities. Due to the existence of nonlinear term, projection technique is not applied to mixed variational inequalities. But when nonlinear term is a proper convex lower semicontinuous functional, we can use resolvent operator technique instead of projection technique to solve mixed variational inequalities. Inspired and motivated by the recent research work, we suggest and analyze three-step iterative methods for finding the common element of the set of fixed points of nonexpansive mapping and the set of solutions of the mixed variational inequalities, we also study the criteria of the new iterative scheme under some mild conditions. Our results presented here are new and interesting and include many previous results as special cases.

\section{Preliminaries and basic results}

Let $\mathrm{K}$ be a nonempty closed and convex set in a real Hilbert space, whose inner product and norm are denoted by $\langle\cdot, \cdot\rangle$ and $\|\cdot\|$, respectively. Let $T: K \rightarrow H$ be a nonlinear mapping and $\mathrm{S}$ be a nonexpansive mapping. Let $F: H \rightarrow R$ be a proper, convex, lower semicontinuous, and nondifferentiable function. We consider the following problem: Find $u \in K$, such that

$$
\langle T u, v-u\rangle+F(v)-F(u) \geq 0, \text { for all } v \in K,
$$

which is called the mixed variational inequalities considered and studied by some authors, see [3-6] and the references therein. We note that if $F$ is the indicator function of a closed convex set $K$ in $H$, that is,

$$
F(u) \equiv I_{K}(u)=\left\{\begin{array}{l}
0, \quad \text { if } u \in K \\
+\infty, \quad \text { otherwise }
\end{array}\right.
$$

then the mixed variational inequality(1)is equivalent to finding $u \in K$, such that

$$
\langle T u, v-u\rangle \geq 0, \text { for all } v \in K,
$$

which is the classical variational inequality problem introduced and studied by Stampacchia [7] in 1964. For the numerical methods for(2), readers may consult the recent state-of-the-art paper [1-9] and references therein. We now recall some well-known concepts and results.

Definition 2.1 If $\mathrm{A}$ is a maximal monotone mapping on $\mathrm{H}$, then for a constant $\rho>0$, the resolvent operator associated with $\mathrm{A}$ is defined by

$$
J_{\rho}^{A}(u)=(I+\rho A)^{-1}(u), \quad \text { for all } u \in H,
$$


where I is the identity operator on $H$. In addition, the resolvent operator is a single-valued and nonexpansive, that is , for all $u, v \in H$,

$$
\left\|J_{\rho}^{A}(u)-J_{\rho}^{A}(v)\right\| \leq\|u-v\| .
$$

Remark 2.1 It is well known that the subdifferential $\partial F$ of a proper, convex, lower semicontinuous, and nondifferentiable function $F: H \rightarrow R$ is a maximal monotone mapping, we denote by

$$
J_{\rho}^{\partial F}(u)=(I+\rho \partial F)^{-1}(u), \text { for all } u \in H .
$$

the resolvent operator associated with $\partial F$, which is defined everywhere on $\mathrm{H}$.

Lemma 2.1 The function $u \in K$ is a solution of the mixed variational inequality (1) if and only if $u \in K$ satisfies the relation

$$
u=J_{\rho}^{\partial F}(I-\rho T)(u) .
$$

It is clear from Lemma 2.1 that the mixed variational inequalities and the fixed point problems are equivalent. This alternative formulation has played a significant role in the studies of the variational inequalities and related optimization problems. Let $S$ be a nonexpansive mapping. We denote the set of the fixed points of $S$ by $G(S)$ and the set of solutions of the mixed variational inequalities (1) by $V I(K, T, F)$. We can characterize the problem. If $x^{*} \in G(S) \cap V I(K, T, F)$, then $x^{*} \in G(S)$ and $x^{*} \in V I(K, T, F)$. Thus from Lemma 2.1, we have

$$
x^{*}=S\left(x^{*}\right)=J_{\rho}^{\partial F}(I-\rho T)\left(x^{*}\right)=S J_{\rho}^{\partial F}(I-\rho T)\left(x^{*}\right),
$$

where $\rho>0$ is a constant.

This fixed point formulation is used to suggest the following iterative methods for finding a common element of two different sets of the fixed points of the nonexpansive mapping and of solutions of the mixed variational inequalities.

Algorithm 2.1 For a given $x_{0} \in K$, compute the sequence $\left\{x_{n}\right\}$ by the iterative scheme

$$
\begin{gathered}
z_{n}=\left(1-c_{n}\right) x_{n}+c_{n} S J_{\rho}^{\partial F}(I-\rho T) x_{n}, \\
y_{n}=\left(1-b_{n}\right) x_{n}+b_{n} S J_{\rho}^{\partial F}(I-\rho T) z_{n}, \\
x_{n+1}=\left(1-a_{n}\right) x_{n}+a_{n} S J_{\rho}^{\partial F}(I-\rho T) y_{n},
\end{gathered}
$$

where $a_{n}, b_{n}, c_{n} \in[0,1]$ for all $n \geq 0$ and $S$ is the nonexpansive mapping. Algorithm 2.1 is a three-step predictor-corrector method. We remark that if $F$ is the indicator function of a closed convex set $K$ in $H$, then the resolvent 
operator $J_{\rho}^{\partial F}=P_{k}$, the projection of $\mathrm{H}$ onto K. Consequently, the Algorithm 2.1 is equivalent to Algorithm 2.1 in Noor and Huang [1]. Note that for $c_{n} \equiv 0$, Algorithm2.1 reduces to:

Algorithm 2.2 For an arbitrarily chosen initial point $x_{0} \in K$, compute the sequence $\left\{x_{n}\right\}$ by the iterative scheme

$$
\begin{gathered}
y_{n}=\left(1-b_{n}\right) x_{n}+b_{n} S J_{\rho}^{\partial F}(I-\rho T) x_{n}, \\
x_{n+1}=\left(1-a_{n}\right) x_{n}+a_{n} S J_{\rho}^{\partial F}(I-\rho T) y_{n},
\end{gathered}
$$

where $a_{n}, b_{n} \in[0,1]$ for all $n \geq 0$ and $\mathrm{S}$ is the nonexpansive mapping. In particular, three-step method is quite general and includes several new and previous known algorithms for solving variational inequalities as special cases.

Definition 2.2 A mapping $T: K \rightarrow H$ is called $\mu$-Lipschitzian, if for all $x, y \in K$, there exists a constant $\mu>0$, such that

$$
\|T x-T y\| \leq \mu\|x-y\| .
$$

Definition 2.3 ([10]) ( A mapping $T: K \rightarrow H$ is called relaxed $(\gamma, r)$ cocoercive if for all $x, y \in K$, there exists constants $\gamma \geq 0, r \geq 0$, such that

$$
\langle T x-T y, x-y\rangle \geq-\gamma\|T x-T y\|^{2}+r\|x-y\|^{2} .
$$

Lemma2.2 ([11]) Suppose $\left\{\delta_{k}\right\}_{k=0}^{\infty}$ is a nonnegative sequence satisfying the following inequality:

$$
\delta_{k+1} \leq\left(1-\lambda_{k}\right) \delta_{k}+\sigma_{k}, k \geq 0
$$

with $\lambda_{k} \in[0,1], \Sigma_{k=0}^{\infty} \lambda_{k}=\infty$ and $\sigma_{k}=o\left(\lambda_{k}\right)$. Then $\lim _{k \rightarrow \infty} \delta_{k}=0$.

\section{Main results}

In this section, we investigate the strong convergence of Algorithms 2.1 and 2.2 in finding the common element of two sets of solutions of the mixed variational inequalities and of fixed points of nonexpansive mapping, which is the main motivation of this paper.

Theorem 3.1 Let $\mathrm{K}$ be a closed convex subset of a real Hilbert space $\mathrm{H}$. Let $\mathrm{T}$ be a relaxed $(\gamma, r)$-cocoercive and $\mu$-Lipschitzian mapping of $\mathrm{K}$ into $\mathrm{H}$, and $\mathrm{S}$ be a nonexpansive mapping of $\mathrm{K}$ into $\mathrm{K}$ such that $G(S) \cap V I(K, T, F) \neq$ $\emptyset$. Let $\left\{x_{n}\right\}$ be a sequence defined by Algorithm 2.1, for any initial point $x_{0} \in K$, if the following conditions hold. 


$$
0<\rho<2\left(r-\gamma \mu^{2}\right) / \mu^{2}, \gamma \mu^{2}<r
$$

$$
a_{n}, b_{n}, c_{n} \in[0,1] \text { and } \Sigma_{n=0}^{\infty} a_{n}=\infty .
$$

Then $\left\{x_{n}\right\}$ obtained from Algorithm 2.1 converges strongly to $x^{*} \in G(S) \cap$ $V I(K, T, F)$.

Proof. $x^{*} \in K$ be a solution of $G(S) \cap V I(K, T, F)$. Then

$$
\begin{gathered}
x^{*}=\left(1-c_{n}\right) x^{*}+c_{n} S J_{\rho}^{\partial F}(I-\rho T) x^{*}, \\
=\left(1-b_{n}\right) x_{*}+b_{n} S J_{\rho}^{\partial F}(I-\rho T) x^{*}, \\
=\left(1-a_{n}\right) x_{*}+a_{n} S J_{\rho}^{\partial F}(I-\rho T) x^{*},
\end{gathered}
$$

where $a_{n}, b_{n}, c_{n} \in[0,1]$ are come constants. To prove the result, we need first to evaluate $\left\|x_{n+1}-x^{*}\right\|$ for all $n \geq 0$.

From (5), (10), and the resolvent operator $J_{\rho}^{\partial F}$ and the nonexpansive mapping $S$, we have

$$
\begin{aligned}
\left\|x_{n+1}-x^{*}\right\|= & \|\left(1-a_{n}\right) x_{n}+a_{n} S J_{\rho}^{\partial F}(I-\rho T) y_{n} \\
& -\left(1-a_{n}\right) x^{*}-a_{n} S J_{\rho}^{\partial F}(I-\rho T) x^{*} \| \\
\leq & \left(1-a_{n}\right)\left\|x_{n}-x^{*}\right\|+a_{n}\left\|S J_{\rho}^{\partial F}(I-\rho T) y_{n}-S J_{\rho}^{\partial F}(I-\rho T) x^{*}\right\| \\
\leq & \left(1-a_{n}\right)\left\|x_{n}-x^{*}\right\|+a_{n}\left\|y_{n}-x^{*}-\rho\left(T y_{n}-T x^{*}\right)\right\| .
\end{aligned}
$$

From the relaxed $(\gamma, r)$-cocoercive and $\mu$-Lipschitzian definition on $\mathrm{T}$,

$$
\begin{aligned}
& \left\|y_{n}-x^{*}-\rho\left(T y_{n}-T x^{*}\right)\right\|^{2} \\
\leq & \left\|y_{n}-x^{*}\right\|^{2}-2 \rho \gamma \mu^{2}\left\|y_{n}-x^{*}\right\|^{2}-2 \rho r\left\|y_{n}-x^{*}\right\|^{2}+\rho^{2} \mu^{2}\left\|y_{n}-x^{*}\right\|^{2} \\
= & \theta^{2}\left\|y_{n}-x^{*}\right\|^{2}
\end{aligned}
$$

where $\theta=\sqrt{1+2 \rho \gamma \mu^{2}-2 \rho r+\rho^{2} \mu^{2}}$. It follows from (6) that $\theta<1$ Combining (11) and (12), we have

$$
\left\|x_{n+1}-x^{*}\right\| \leq\left(1-a_{n}\right)\left\|x_{n}-x^{*}\right\|+a_{n} \theta\left\|y_{n}-x^{*}\right\| .
$$

From (4), (9) and the nonexpansivity of the mapping $S$ and $J_{\rho}^{\partial F}$, we have

$$
\begin{aligned}
\left\|y_{n}-x^{*}\right\| & \leq\left(1-b_{n}\right)\left\|x_{n}-x^{*}\right\|+b_{n}\left\|S J_{\rho}^{\partial F}(I-\rho T) z_{n}-S J_{\rho}^{\partial F}(I-\rho T) x^{*}\right\| \\
& \leq\left(1-b_{n}\right)\left\|x_{n}-x^{*}\right\|+b_{n}\left\|z_{n}-x^{*}-\rho\left(T z_{n}-T x^{*}\right)\right\| .
\end{aligned}
$$

Now from the relaxed $(\gamma, r)$-cocoercive and $\mu$-Lipschitzian definition on $T$, it yields that

$$
\begin{aligned}
& \left\|z_{n}-x^{*}-\rho\left(T z_{n}-T x^{*}\right)\right\|^{2} \\
\leq & \left\|z_{n}-x^{*}\right\|^{2}-2 \rho \gamma \mu^{2}\left\|z_{n}-x^{*}\right\|^{2}-2 \rho r\left\|z_{n}-x^{*}\right\|^{2}+\rho^{2} \mu^{2}\left\|z_{n}-x^{*}\right\|^{2} \\
= & \theta^{2}\left\|z_{n}-x^{*}\right\|^{2} .
\end{aligned}
$$


In a similar way, from (3) and (8), it follows that

$\left\|z_{n}-x^{*}\right\| \leq\left(1-c_{n}\right)\left\|x_{n}-x^{*}\right\|+c_{n} \theta\left\|x_{n}-x^{*}\right\|=\left(1-c_{n}(1-\theta)\right)\left\|x_{n}-x^{*}\right\| \leq\left\|x_{n}-x^{*}\right\|$.

Then from (14), (15) and (16)

$\left\|y_{n}-x^{*}\right\| \leq\left(1-b_{n}\right)\left\|x_{n}-x^{*}\right\|+b_{n} \theta\left\|z_{n}-x^{*}\right\| \leq\left(1-b_{n}\right)\left\|x_{n}-x^{*}\right\|+b_{n} \theta\left\|x_{n}-x^{*}\right\|$.

From (13) and (17) that

$$
\begin{aligned}
\left\|x_{n+1}-x^{*}\right\| & \leq\left(1-a_{n}\right)\left\|x_{n}-x^{*}\right\|+a_{n} \theta\left[\left(1-b_{n}\right)\left\|x_{n}-x^{*}\right\|+b_{n} \theta\left\|x_{n}-x^{*}\right\|\right] \\
& \leq\left[1-\theta(1-\theta) a_{n}\right]\left\|x_{n}-x^{*}\right\| .
\end{aligned}
$$

Letting $\delta_{k}=\left\|x_{k}-x^{*}\right\|, \lambda_{k}=\theta(1-\theta) a_{n}, \sigma_{k}=0$. It follows from (18), the condition (7) and Lemma 2.2 that $\lim _{n \rightarrow \infty}\left\|x_{n}-x^{*}\right\|=0$, i.e., $\left\{x_{n}\right\}$ strongly converges to $x^{*}$. This is completed the proof.

If $c_{n} \equiv 0$, then the following result is a special case of Theorem 3.1.

Theorem 3.2 Let $\mathrm{K}$ be a closed convex subset of a real Hilbert space $\mathrm{H}$. Let $\mathrm{T}$ be a relaxed $(\gamma, r)$-cocoercive and $\mu$-Lipschitzian mapping of $\mathrm{K}$ into $\mathrm{H}$, and $\mathrm{S}$ be a nonexpansive mapping of $\mathrm{K}$ into $\mathrm{K}$ such that $G(S) \cap V I(K, T, F) \neq$ $\emptyset$. Let $\left\{x_{n}\right\}$ be a sequence defined by Algorithm 2.2, for any initial point $x_{0} \in K$, with conditions $0<\rho<2\left(r-\gamma \mu^{2}\right) / \mu^{2}, \gamma \mu^{2}<r, a_{n}, b_{n} \in[0,1]$ and $\sum_{n=0}^{\infty} a_{n}=\infty$, then $\left\{x_{n}\right\}$ obtained from Algorithm 2.2 converges strongly to $x^{*} \in G(S) \cap V I(K, T, F)$.

Remark 3.1 Theorems 3.1 and 3.2 extend the corresponding results of [12] and include many previous corresponding results as special cases.

\section{References}

1. M.A. Noor, Zhenyu Huang, Three-step methods for nonexpansive mappings and variational inequalities, Appl. Math. Comput. (2006), doi: 10.1016/J.ams. 2006. 08. 088.

2. M. A. Noor, Mixed variational inequalities, Appl. Math. Lett., 3(2)1990: 73-75.

3. M. A. Noor, A new iterative method for monotone mixed inequalities, Math. Comput. Modelling, 26(7) 1997: 29-34.

4. Zhenyu Huang, M. Aslam Noor, Strong convergence citeria for nonexpansive and related $(\gamma, r)$-cocoercive mappings in Hilbert spaces,Preprint, 2006.

5. M. Aslam Noor, Some Algorithm for general monotone mixed variational inequalities, Math. Comput. Modell. 29(1999): 1-9. 
6. M. Aslam Noor, New approximation schemes for general variational inequalities, J. Math. Anal. Appl. 251(2000): 217-229.

7. G. Stampacchia, Formes bilineaires coercivites sur les ensembles convexes,Comptes Rendus de 1' Acdemie des Sciences. Paris 258(1964): 44134416.

8. W. Takahashi, M. Toyoda, Weak convergence theorems for nonexpansive mappings and monotone mappings, J.Optim.theoriy Appl. 118(2)(2003): 417-428.

9. R.U. Verma, Generalized system for relaxed cocoercive variational inequalities and projection methods, J.Optim.Theory Appl. 121(1)(2004): 203-210.

10. X.L. Weng, Fixed point iteration for local strictly pscudocontractive mappings, Proc. Amer. Math. Soc.113(1991): 727-731.

Received: June 27, 2007 